\section{Rabies Acquired through Mucosal Exposure, China, 2013}

\author{
Hong Zhao, Jian Zhang, Cong Cheng, \\ Yi-Hua Zhou
}

Author affiliations: Second Hospital of Nanjing at Southeast University, Nanjing, China (H. Zhao, J. Zhang, C. Cheng); Nanjing University Medical School, Nanjing (Y.-H. Zhou)

DOI: https://doi.org/10.3201/eid2505.181413

In China in 2013, a man acquired rabies after sucking wounds of his son, who had been bitten by a stray dog. The man declined postexposure prophylaxis (hyperimmunoglobulin and vaccine) and died; the son accepted prophylaxis and survived. Physicians should be aware of rabies transmission through mucosal exposure and encourage postexposure prophylaxis.

$\mathrm{R}$ abies, caused by the rabies virus (family Rhabdoviridae, genus Lyssavirus), is a zoonotic encephalitis with a mortality rate of almost $100 \%$. Although rare in industrialized countries because of mandatory vaccination of dogs and other domestic pets, rabies frequently occurs in developing countries (1). In industrialized countries, the virus is transmitted to humans mostly by contact with wild animals; in developing countries, by dog bites (1). We report a fatal case of rabies in a man who had sucked the wounds of his son who had been bitten by a stray dog. The study was approved by the institutional ethics review committees of the Second Hospital of Nanjing and Nanjing Drum Tower Hospital, Nanjing, China, and was performed in accordance with the ethics standards in the 1964 Declaration of Helsinki and its later amendments. Written informed consent was obtained from the patient's son.

The patient was a 41-year-old man from a village in northern Jiangsu Province, China. On August 19, 2013, the man's 17-year-old son was bitten on the left leg by a stray dog, leaving bleeding wounds on the lower part of the gastrocnemius muscles. Because of concern about rabies, the man and other family members immediately washed the boy's wounds with water, and the man's neighbors killed and buried the stray dog. While washing his son's wounds, the man made a dramatic scene by crouching down to suck "toxic blood" from his son's wounds and spitting out the blood several times. He then sent his son to a local hospital, where the physicians immediately (within 1 hour after the dog bite) cleaned the wounds by using standard procedures and administered postexposure prophylaxis (rabies hyperimmunoglobulin and the first dose of freeze-dried rabies vaccine for human use [Vero cells]; Liaoning Chengda Biotechnology, http:// www.cdbio.cn). The son received another 4 doses of rabies vaccine on days $3,7,14$, and 28 after the bite. Because the man had sucked the wounds, the physicians also recommended that he receive postexposure prophylaxis (rabies hyperimmunoglobulin and vaccine); however, the man declined because of concern over the relatively high cost and because he considered that he had spat out all the sucked blood and could not have "so bad luck." Thus, the man did not receive any medical treatment.

On September 23, 2013, the man was referred to the Nanjing Second Hospital, the main infectious diseases hospital in Jiangsu, with a 2-day history of general malaise, sleep disturbance, waking in a cold sweat, irritability to air and sound, involuntary contraction of pharyngeal muscles, and choking when drinking and eating. At the time of examination, the patient showed remarkable agitation, delirium, and hallucination. Saliva was collected and tested for rabies viral RNA by reverse transcription PCR; the result was positive for rabies virus. The patient received systemic support and symptomatic therapy but died on September 24. The clinical course of rabies in this patient progressed rapidly; although the incubation period for this patient was 33 days (in agreement with incubation period of 20-60 days for most patients), he died 3 days after the appearance of furious (classical) symptoms. The patient's son remained in good health 5 years later.

The main route for rabies virus transmission is bites of infected dogs and other animals. Unusual transmission routes include mucosal exposure, aerosol inhalation, organ transplantation (2-7), and contamination of broken skin by blood from wounds of a person bitten by an infected $\operatorname{dog}(8)$. The most effective way to control rabies in humans is mandatory vaccination of all dogs and other domestic pets, but widespread vaccination is not feasible in China and resource-limited countries. Although China's central and local governments recommend that all domestic pets be vaccinated against rabies, implementation of vaccination is difficult in many rural areas of China. Thus, for preventing rabies in humans in rabies-endemic countries and regions, postexposure prophylaxis is crucial.

The rabies case that we report was acquired after sucking wounds created by the bite of a presumably rabies-infected dog. Evidently, the virus invaded the patient through his oral mucosa. Unfortunately, the patient declined postexposure prophylaxis and died.

This case underscores the possibility of rabies transmission by mucosal exposure, which should be made widely known to the public. To further reduce this disease in 
rabies-endemic countries, physicians should emphasize the high likelihood of transmission of rabies virus after mucosal exposure and try to persuade persons at risk to receive postexposure prophylaxis.

\section{About the Author}

Dr. Zhao is a chief clinician specializing in the diagnosis and treatment of infectious diseases at the Department of Infectious Diseases, Second Hospital of Nanjing. Her research interests include viral hepatitis and other viral diseases.

\section{References}

1. Etheart MD, Kligerman M, Augustin PD, Blanton JD, Monroe B, Fleurinord L, et al. Effect of counselling on health-care-seeking behaviours and rabies vaccination adherence after dog bites in Haiti, 2014-15: a retrospective follow-up survey. Lancet Glob Health. 2017;5:e1017-25. http://dx.doi.org/10.1016/ S2214-109X(17)30321-2

2. Kan VL, Joyce P, Benator D, Agnes K, Gill J, Irmler M, et al. Risk assessment for healthcare workers after a sentinel case of rabies and review of the literature. Clin Infect Dis. 2015;60:341-8. http://dx.doi.org/10.1093/cid/ciu850

3. Srinivasan A, Burton EC, Kuehnert MJ, Rupprecht C, Sutker WL, Ksiazek TG, et al.; Rabies in Transplant Recipients Investigation Team. Transmission of rabies virus from an organ donor to four transplant recipients. N Engl J Med. 2005;352:1103-11. http://dx.doi.org/10.1056/NEJMoa043018

4. Vora NM, Basavaraju SV, Feldman KA, Paddock CD, Orciari L, Gitterman S, et al.; Transplant-Associated Rabies Virus Transmission Investigation Team. Raccoon rabies virus variant transmission through solid organ transplantation. JAMA. 2013;310:398-407. http://dx.doi.org/10.1001/jama.2013.7986

5. Ross RS, Wolters B, Hoffmann B, Geue L, Viazov S, Grüner N, et al. Instructive even after a decade: complete results of initial virological diagnostics and re-evaluation of molecular data in the German rabies virus "outbreak" caused by transplantations. Int J Med Microbiol. 2015;305:636-43. http://dx.doi.org/10.1016/ j.ijmm.2015.08.013

6. Zhou H, Zhu W, Zeng J, He J, Liu K, Li Y, et al. Probable rabies virus transmission through organ transplantation, China, 2015. Emerg Infect Dis. 2016;22:1348-52. http://dx.doi.org/10.3201/ eid2208.151993

7. Chen S, Zhang H, Luo M, Chen J, Yao D, Chen F, et al. Rabies virus transmission in solid organ transplantation, China, 20152016. Emerg Infect Dis. 2017;23:1600-2. http://dx.doi.org/ 10.3201/eid2309.161704

8. Zhu JY, Pan J, Lu YQ. A case report on indirect transmission of human rabies. J Zhejiang Univ Sci B. 2015;16:969-70. http://dx.doi.org/10.1631/jzus.B1500109

Address for correspondence: Yi-Hua Zhou, Nanjing Drum Tower Hospital, Departments of Laboratory Medicine and Infectious Diseases, 321 Zhongshan Rd, Nanjing 210008, China; email: zgr03summer@126.com

\section{Endemic Severe Fever with Thrombocytopenia Syndrome, Vietnam}

\author{
Xuan Chuong Tran, Yeojun Yun, Le Van An, \\ So-Hee Kim, Nguyen T. Phuong Thao, \\ Phan Kim C. Man, Jeong Rae Yoo, \\ Sang Taek Heo, Nam-Hyuk Cho, Keun Hwa Lee
}

Author affiliations: The Hue University Hospital and Hue University of Medicine and Pharmacy, Hue, Vietnam (X.C. Tran, L.V. An, N.T.P. Thao, P.K.C. Man); Ewha Womans University, Seoul, South Korea (Y. Yun); The Kyung Hee University, Seoul (S.-H. Kim); The Jeju National University College of Medicine, Jeju, South Korea (J.R. Yoo, S.T. Heo, K.H. Lee); Seoul National University College of Medicine, Seoul (N.-H. Cho)

DOI: https://doi.org/10.3201/eid2505.181463

Severe fever with thrombocytopenia syndrome (SFTS), a tickborne viral disease, has been identified in China, South Korea, and Japan since 2009. We found retrospective evidence of SFTS virus (SFTSV) infection in Vietnam, which suggests that SFTSV infections also occur in Vietnam, where the virus has not been known to be endemic.

Severe fever with thrombocytopenia syndrome virus (SFTSV) is a tickborne virus (genus Phlebovirus, family Phenuiviridae) that can cause a mild to severe febrile illness similar to hemorrhagic fever (1). Phleboviruses have been found in the Americas, Asia, Africa, and the Mediterranean region. For example, Heartland virus (HRTV), another tickborne phlebovirus, was identified in northwestern Missouri, USA, in 2009 (2). Malsoor virus, a new bat phlebovirus closely related to SFTSV and HRTV, was identified in western India, and a phlebovirus similar to SFTSV and HRTV was isolated from ticks in Australia $(3,4)$.

Severe fever with thrombocytopenia syndrome (SFTS) illness was first confirmed in China in 2009. It was retrospectively identified in South Korea in 2010 and the western regions of Japan in $2013(1,5,6)$. SFTS is characterized by acute high fever, thrombocytopenia, leukopenia, elevated serum hepatic enzymes, gastrointestinal symptoms, and multiorgan failure and has a death rate of $16.2 \%-30 \%$ $(1,6,7)$. Atypical signs and symptoms and asymptomatic infections also have been identified $(5,8)$. Most SFTSV infections occur through Haemaphysalis longicornis ticks, although SFTSV transmission can also occur through close contact with an infected patient (8).

To investigate evidence of SFTSV infections in Vietnam, we collected serum samples from 80 patients with acute febrile illnesses admitted to Hue University Hospital (Hue, Vietnam) 